\title{
ON REGULAR SEQUENCES
}

\section{J. DIEUDONNÉ}

In memory of TAdasi NakAyama

1. The concept of regular sequence of elements of a ring $A$ (first introduced by Serre under the name of $A$-sequence [2]), has far-reaching uses in the theory of local rings and in algebraic geometry. It seems, however, that it loses much of its importance when $A$ is not a noetherian ring, and in that case, it probably should be superseded by the concept of quasi-regular sequence [1].

One of the convenient properties of a regular sequence $t_{1}, \ldots, t_{n}$ in a noetherian local ring $A$, where the $t_{i}$ 's belong to the maximal ideal of $A$, is that the sequence remains regular after an arbitrary permutation of its terms. This is due to the fact that in such a case, the notions of regular sequence and of quasi-regular sequence coincide $[1,15.1 .10]$, and the notion of quasjregular sequence is independent of the order of the elements of the sequence.

I will give below an example of a non noetherian local ring $A$ and of two elements $t_{1}, t_{2}$ of the maximal ideal of $A$, such that the sequence $\left(t_{1}, t_{2}\right)$ is regular, whilst the sequence $\left(t_{2}, t_{1}\right)$ is not. Such unpleasant phenomena greatly reduce the usefulness of the notion of regular sequence.

2. To construct our example, we start with the ring $B$ of all germs of indefinitely differentiable functions of a real variable $x$ in the neighborhood of 0 . It is well known that $B$ is a local ring, whose maximal ideal $\mathfrak{n}$ is generated by the germ $i$ of the identity mapping $x \rightarrow x$; the intersection of all powers $\mathfrak{n}^{k}(k=1,2, \ldots)$ is the ideal $\mathfrak{r} \neq 0$ consisting of all germs of functions whose derivatives all vanish at $x=0$. Observe that the complement of $\mathfrak{r}$ in $B$ consists of regular elements of $B$ (i.e. elements which are not zero-divisors).

Now consider the ring of polynomials $B[T]$ in one indeterminate, and let $C$ be the quotient of $B[T]$ by the ideal $\mathfrak{r} T B[T]$, consisting of all polynomials $r_{1} T+\cdots+r_{m} T^{m}$ having their coefficients in $\dot{r}$. We prove that in $C$, the

Received June 26, 1965. 
sequence consisting of the classes $s_{1}, s_{2}$ of $i$ and $T$ respectively, is regular. Indeeed we have $\mathfrak{r} T B[T] \subset i B[T]=\mathfrak{n} B[T]$; furthermore the relation if $\in \mathfrak{r} T B[T]$ for a polynomial $f \in B[T]$ means that if $f[T]=c_{0}+c_{1} T+\cdots+c_{m} T^{m}$, then $i c_{0}=0$ and $i c_{k} \in \mathfrak{r}$ for $k \geqslant 1$; but this implies that $c_{0}=0$, and $c_{k} \in \mathfrak{r}$ for $k \geqslant 1$, by definition of $B$. Thus we see that $s_{1}$ is a regular element of $C$; furthermore we have $C / s_{1} C=B[T] / i B[T]=\mathbf{R}[T]$, since $B / i B=B / \mathfrak{n}=\mathbf{R}$ (real field); as $\mathbf{R}[T]$ is an integral domain, the image of $s_{2}$ in $C / s_{1} C$, which is identified with $T$ in $\mathbf{R}[T]$, is a regular element, and this shows that the sequence $\left(s_{1}, s_{2}\right)$ is regular.

On the other hand, by definition $s_{2}$ is a zero-divisor in $C$, for the images of the elements of $\mathfrak{r}$ (other than 0 ) in $C$ are $\neq 0$, but the images of their products by $T$ are all $0 . A$ fortiori the sequence $\left(s_{2}, s_{1}\right)$ is not regular.

To form our example, note that the ideal $s_{1} C+s_{2} C$ is a maximal ideal $\mathrm{m}$; one has only to take for $A$ the local ring $C_{\mathfrak{m}}$, for $t_{1}$ and $t_{2}$ the images in $A$ of $s_{1}$ and $s_{2}$; it is readily verified that the elements of the complement of $m$ in $C$ are not zero-divisors, hence $t_{2}$ is a zero-divisor in $A$; on the other hand, the sequence $\left(t_{1}, t_{2}\right)$ is regular by flatness.

3. The same construction gives an example of a quasi-regular element of $C$ which is not regular, namely the element $s_{2}$ (a regular sequence is quasiregular, and a subsequence of a quasi-regular sequence is quasi-regular [1, 15. 1. 10 and 15. 1.9]).

\section{BIBLIOGRAPHY}

[1] A. Grothendieck, Eléments de Géométrie algébrique, chap. IV, 1re partie, Publ. math. I.H.E.S. No. 20, 1964.

[2] J. P. Serre, Sur la dimension cohomologique des anneaux et des modules noethériens, Proc. Intern. Symp. on Alg. Number theory, p. 176-189, Tokyo-Nikko, 1955.

\section{University of Nice}

Nice, France 